\title{
Evolution of the Initially Recruited SHARE Panel Sample Over the First Six Waves
}

\author{
Sabine Friedel ${ }^{1}$ and Tim Birkenbach ${ }^{2}$
}

\begin{abstract}
Attrition is a frequently observed phenomenon in panel studies. The loss of panel members over time can hamper the analysis of panel survey data. Based on data from the Survey of Health, Ageing and Retirement in Europe (SHARE), this study investigates changes in the composition of the initially recruited first-wave sample in a multi-national face-to-face panel survey of an older population over waves. By inspecting retention rates and R-indicators, we found that, despite declining retention rates, the composition of the initially recruited panel sample in Wave 1 remained stable after the second wave. Thus, after the second wave there is no further large decline in representativeness with regard to the first wave sample. Changes in the composition of the sample after the second wave over time were due mainly to mortalityrelated attrition. Non-mortality-related attrition had a slight effect on the changes in sample composition with regard to birth in survey country, area of residence, education, and social activities. Our study encourages researchers to investigate further the impact of mortality- and non-mortality-related attrition in multi-national surveys of older populations.
\end{abstract}

Key words: R-indicator; wave nonresponse; mortality- and non-morality-related attrition; panel sample composition.

\section{Introduction}

Panel surveys of older populations in Europe have become the focus of widespread interest in recent decades. Falling fertility rates (Myrskylä et al. 2013) and greater life expectancy (Leon 2011) bring many challenges for Western European societies. To investigate these dynamic processes, researchers need data that allow them to provide evidence of changes over time (Olsen 2018). In contrast to cross-sectional surveys, panel surveys fulfil this

1 University of Mannheim, SFB 884 "Political Economy of Reforms", B6, 30-32, 68131 Mannheim, Germany. Email: s.friedel@uni-mannheim.de

${ }^{2}$ Max Planck Institute for Social Law and Social Policy, Munich Center for the Economics of Aging (MEA), Amalienstrasse 33, 80799 Munich, Germany. Email: birkenbach@mea.mpisoc.mpg.de

Acknowledgments: Funded by the Deutsche Forschungsgemeinschaft (DFG, German Research Foundation) Project-ID 139943784 - SFB 884. This article uses data from SHARE Wave 1 (DOI: https://doi.org/10.6103/SHARE.w1.600). The SHARE data collection has been primarily funded by the European Commission through FP5 (QLK6-CT-2001-00360), FP6 (SHARE-I3: RII-CT-2006-062193, COMPARE: CIT5-CT-2005-028857, SHARELIFE: CIT4-CT-2006-028812) and FP7 (SHARE-PREP: $\mathrm{N}^{\circ} 211909$, SHARE-LEAP: $\mathrm{N}^{\circ} 227822$, SHARE M4: $\mathrm{N}^{\circ}$ 261982). Additional funding from the German Ministry of Education and Research, the Max Planck Society for the Advancement of Science, the U.S. National Institute on Aging (U01_AG09740-13S2, P01_AG005842, P01_AG08291, P30_AG12815, R21_AG025169, Y1-AG-4553-01, IAG_BSR06-11, OGHA_04-064, HHSN271201300071C) and from various national funding sources is gratefully acknowledged (see www.share-project.org). The authors would like to gratefully thank Annette Scherpenzeel for her ideas, exceptional support, and feedback, Michael Bergmann, Thorsten Kneip, Peter Lugtig, and Annelies Blom for their advice and feedback, and Thomas Klausch for his advice and expertise on R-indicators. 
requirement because they repeatedly collect data from the same respondents over time (Lynn 2009).

However, a major detracting feature of panel surveys is the risk of attrition - that is, the loss of panel members from the initially recruited sample over time (Binder 1998). Panel attrition is a frequent phenomenon that has been observed during the last decades (Fitzgerald et al. 1998; Watson 2003; Buck et al. 2006). Attrition may occur because panel members are no longer able or willing to participate or because they can no longer be located or contacted (Lynn and Lugtig 2017). The largest amount of drop out occurs in the second wave (Watson and Wooden 2009; Schoeni et al. 2013). When attrition occurs, changes over time cannot be observed from the beginning to the end of the panel because one measure is missing in two consecutive waves (Lynn and Lugtig 2017). This absence of data can lead to restrictions when researchers want to analyze changes in the data. Thus, we need to inform researchers about attrition in the data they use.

Particularly in panel surveys of older populations, researchers are faced with a greater risk of attrition due to death. In an investigation of characteristics associated with attrition in the English Longitudinal Study of Ageing (ELSA) and the U.S. Health and Retirement Study (HRS), Banks et al. (2011) found that the mortality rate between two waves among panel members aged 70-80 years was 15\%, and that among 55-64 year-old panel members it was 4\%. In contrast, for the Panel Study of Income Dynamics (PSID), which is a household panel survey, Watson (2003) reported a mortality rate of only $0.5 \%$ between two waves. Thus, the risk of mortality-related attrition is much higher in panel surveys of older populations compared to those that collect data on younger populations.

Deaths in panel surveys of older populations are not problematic per se. Older populations are not fixed, and all older populations are affected by deaths (Smith et al. 2009). Deaths occur both in the population and in the sample, and thus deaths of panel members change the composition of the data sample and of the population about which researchers want to draw conclusions. In both settings - the population and the sample individuals who have a lower risk of dying, for example because they have a high socioeconomic and health status, are more likely to survive to old age than individuals with a low socioeconomic and health status (Banks et al. 2011). Thus, we assume that mortality in panel surveys of older populations is selective. However, deaths reflect changes in the composition of the population to which the data refer, and, as Smith et al. $(2009,29)$ noted, "as long as these [deaths] can be identified and distinguished from nonresponse, they are easily incorporated in analyses by using a code for dead units."

In contrast to mortality-related attrition, respondents who drop out for other reasons are still present in the population, and their non-participation changes only the composition of the sample. Changes in these individuals' outcomes of interest can no longer be observed in the survey data, although they are occurring in the population. However, this type of attrition is not problematic per se, either, unless it is selective, and thus can affect the validity and interpretation of estimates (Watson and Wooden 2019).

The present study focuses on the Survey of Health, Ageing and Retirement in Europe (SHARE) (Börsch-Supan et al. 2013), a biennial panel study based on people in Europe aged 50 years and older. With its harmonized collection of data in many European countries, SHARE is unique and offers many opportunities to analyze dynamic processes in the European societies. Although previous research has shown that attrition occurs in the 
SHARE panel (Bergmann et al. 2019), little research has investigated in more detail the changes in the composition of the initially recruited panel sample over time (e.g., Bristle et al. 2019). Moreover, little is known about the relation between attrition and the changes in the panel composition over waves when mortality is particularly considered. Both aspects can inform researchers about the impact of attrition on the evolution of the SHARE panel.

To obtain a clear picture of how the composition of the SHARE panel has evolved over waves, we define two samples of interest:

- A: the initially recruited SHARE sample (i.e., the sample first interviewed in Wave 1), and

- B: the initially recruited SHARE sample, excluding respondents who were reported to have died.

Whereas Sample A is fixed over waves and includes all respondents who dropped out, Sample B is dynamic over waves and excludes for each wave separately respondents who were reported to have died before the corresponding wave started. For instance, Sample B in Wave 2 is based on the initially recruited SHARE sample, excluding respondents who were reported to have died before the second wave started, or Sample B in Wave 3 is based on the initially recruited SHARE sample, excluding respondents who were reported to have died before the third wave started. Thus, Sample A investigates total attrition (nonmortality-related and mortality-related), whereas Sample B investigates non-mortalityrelated attrition only.

With these two definitions of the samples of interest, we aim to answer the following research questions:

1. How has the initially recruited first-wave sample (A and B) evolved over the survey waves?

2. Has the evolution of the initially recruited first-wave sample (A and B) over waves varied across countries?

3. What variables/characteristics have played the most important role in the evolution over waves of the sample that excludes reported deaths (Sample B)?

The remainder of this article is organized as follows: In the next section, we describe our SHARE dataset and the variables considered in our analyses. We then answer Research Questions 1 and 2 by applying two aggregate-level measures (retention rates, Rindicators). In Section 4, we apply two variable-level measures (subgroup retention rates, logistic regressions) to answer Research Question 3. Thus, the methods and results for the first two research questions and the methods and results for the third question are presented separately. The article concludes with a summary of the findings and discussion for all three research questions.

\section{Data and Variables}

\subsection{Data}

We used data from the Survey of Health, Ageing and Retirement in Europe (SHARE) (Börsch-Supan 2017). SHARE is a biennial multidisciplinary, cross-national panel survey 
that collects microdata on the health, socio-economic status, and social and family networks of individuals aged 50 years and older and of their partners, regardless of their age. The target persons and their partners are interviewed face-to-face using computerassisted personal interviewing (CAPI) (Börsch-Supan et al. 2013). The first wave of SHARE was conducted in 2004 in eleven European countries and in Israel. Samples from each country are based on a probability sample that is representative of the non-institutionalized population aged 50 years and older (De Luca et al. 2013). The initial individual response rates (RR1, American Association for Public Opinion Research, AAPOR 2016) ranged between $27.9 \%$ and 58.8\% (Bergmann et al. 2019).

For our analyses, we used the first-wave data about respondents' individual and household characteristics and supplemented these data with information about whether or not the respondents had participated in later waves. We restricted our sample to countries that participated in all six observed waves. This selection criterion reduced the sample to nine countries (Austria, Belgium, Denmark, France, Germany, Italy, Spain, Sweden, and Switzerland). Moreover, we restricted our sample to respondents aged 50 years or older. Together, these restrictions decreased the sample to 21,227 panel respondents (Table 1, Respondents aged $50+$ ). About $5 \%$ of the respondents could not be considered because they did not know or refused to report the answer to questions that were used to measure variables included in the analyses. As a consequence, the first analysis sample of Sample A consisted of 20,236 respondents. The sample size by country ranged from 898 in Switzerland to 3,521 in Belgium (see Table 1, Analysis Sample A).

To study further non-mortality-related attrition, we excluded respondents who were reported to have died before a given wave. This exclusion resulted in a dynamic Analysis Sample B (see Table 1, Analysis Sample B, Wave 1-Wave 6). However, the quality of information we used to identify deaths differs between countries. This is due mainly to the fact that most European countries lack a national mortality register or similar records. Therefore, SHARE cannot reliably ascertain the vital status of nonrespondents who drop out because they cannot be located or contacted or because they refuse to be re-interviewed (Bergmann et al. 2019). Thus, the dynamic Analysis Sample B may include unreported deaths.

Table 1. Sample selection of initially recruited first-wave SHARE respondents.

\begin{tabular}{|c|c|c|c|c|c|c|c|c|}
\hline \multirow[b]{2}{*}{ Country } & \multirow{2}{*}{$\begin{array}{c}\begin{array}{c}\text { Respondents } \\
\text { aged } 50+\end{array} \\
\text { Wave } 1\end{array}$} & \multirow{2}{*}{$\begin{array}{c}\begin{array}{c}\text { Analysis } \\
\text { sample A }\end{array} \\
\text { Wave } 1-6\end{array}$} & \multicolumn{6}{|c|}{ Analysis sample B } \\
\hline & & & Wave 1 & Wave 2 & Wave 3 & Wave 4 & Wave 5 & Wave 6 \\
\hline Austria & 1,516 & 1,487 & 1,487 & 1,442 & 1,361 & 1,287 & 1,213 & 1,174 \\
\hline Belgium & 3,631 & 3,521 & 3,521 & 3,474 & 3,356 & 3,237 & 3,120 & 3,017 \\
\hline Denmark & 1,597 & 1,527 & 1,527 & 1,480 & 1,390 & 1,310 & 1,220 & 1,134 \\
\hline France & 2,955 & 2,706 & 2,706 & 2,650 & 2,519 & 2,428 & 2,298 & 2,221 \\
\hline Germany & 2,909 & 2,768 & 2,768 & 2,718 & 2,648 & 2,545 & 2,508 & 2,486 \\
\hline Italy & 2,495 & 2,406 & 2,406 & 2,353 & 2,268 & 2,189 & 2,081 & 1,984 \\
\hline Spain & 2,232 & 2,075 & 2,075 & 1,984 & 1,884 & 1,769 & 1,655 & 1,547 \\
\hline Sweden & 2,961 & 2,848 & 2,848 & 2,778 & 2,640 & 2,486 & 2,349 & 2,268 \\
\hline Switzerland & 931 & 898 & 898 & 882 & 860 & 839 & 816 & 788 \\
\hline Total & 21,227 & 20,236 & 20,236 & 19,761 & 18,926 & 18,090 & 17,260 & 16,619 \\
\hline
\end{tabular}




\subsection{Variables}

Investigating the evolution of the SHARE panel offered the possibility of including a rich set of variables in the models. To examine the evolution of the panel, we selected 23 first-wave key variables from the areas of demographics, social embeddedness, health, and economics, and three survey-specific variables of the questionnaire design (Table 2).

Table 2. Operationalization of information used to examine the evolution of the SHARE panel.

\begin{tabular}{|c|c|}
\hline Variable & Operationalization \\
\hline \multicolumn{2}{|l|}{ Demographics } \\
\hline Gender & $0:$ male; 1 : female \\
\hline Age & $\begin{array}{l}\text { 1: } 50-59 \text { years; } 2: 60-69 \text { years; } 3: 70-79 \text { years; } \\
\text { 4: } 80+\text { years }\end{array}$ \\
\hline Born in survey country & 1: yes; $0:$ no \\
\hline Education level & 1: low; 2 : medium and other; 3 : high \\
\hline Household $(\mathrm{HH})$ size & 1: 1-person $\mathrm{HH} ; 2$ : 2-person $\mathrm{HH} ; 3: 3+$-person $\mathrm{HH}$ \\
\hline Partner in $\mathrm{HH}$ & $0:$ no; $1:$ yes \\
\hline Area of residence & 1: city/large town; 2 : small town; 3 : rural village \\
\hline \multicolumn{2}{|l|}{ Social embeddedness variables } \\
\hline $\begin{array}{l}\text { Residential proximity } \\
\text { of child(ren) }\end{array}$ & $\begin{array}{l}\text { 1: no children; } 2 \text { : child living in household; } 3 \text { : child } \\
\text { living }=1 \mathrm{~km} \text { away; } 4 \text { : child living }>1 \mathrm{~km} \text { away }\end{array}$ \\
\hline Social activities & 0 : no activities; 1 : at least one activity \\
\hline Received help from others & $0:$ no; $1:$ yes \\
\hline Gave help to others & $0:$ no; $1:$ yes \\
\hline \multicolumn{2}{|l|}{ Health variables } \\
\hline Health status & $0:$ good or better; $1:$ fair or poor \\
\hline Chronic diseases & $0:$ none; $1:$ at least one chronic disease \\
\hline Depression (Euro-D) & $\begin{array}{l}\text { 0: no or insufficient symptoms; } 1: 4 \text { or more } \\
\text { depressive symptoms }\end{array}$ \\
\hline Maximum grip strength & $\begin{array}{l}\text { 1: item nonresponse; } 2: 1 \text { st quartile; } 3: 2 \text { nd quartile; } \\
\text { 4: 3rd quartile; } 5: 4 \text { th quartile }\end{array}$ \\
\hline Memory recall ability & $\begin{array}{l}0 \text { : recalled less than half of the words; } 1 \text { : recalled } \\
\text { more than half of the words }\end{array}$ \\
\hline $\begin{array}{l}\text { Hospital overnight stays } \\
\text { in last } 12 \text { months }\end{array}$ & $0:$ no; $1:$ yes \\
\hline Currently smoking & $0:$ no; $1:$ yes \\
\hline Currently drinking & $\begin{array}{l}\text { 0: never; } 1 \text { : less than once a week; } 2: 1-6 \text { times a } \\
\text { week; } 3 \text { : daily }\end{array}$ \\
\hline $\begin{array}{l}\text { Limitation of instrumental } \\
\text { activities of daily living } \\
\text { (IADL) }\end{array}$ & 0: no IADL limitation; 1 : at least one IADL limitation \\
\hline \multicolumn{2}{|l|}{ Economic variables } \\
\hline Employment status & 1: retired; 2 working; 3 : not working and other \\
\hline Make ends meet & 0 : difficulties; 1 : no difficulties \\
\hline Total household income & $\begin{array}{l}\text { 1: item nonresponse; } 2: \text { st quartile; } 3: 2 \text { nd quartile; } \\
\text { 4: } 3 \text { rd quartile; } 5: 4 \text { th quartile }\end{array}$ \\
\hline \multicolumn{2}{|l|}{ Interview process variables } \\
\hline Financial respondent & $0:$ no; 1 : yes \\
\hline Family respondent & $0:$ no; $1:$ yes \\
\hline Household respondent & $0:$ no; 1 : yes \\
\hline
\end{tabular}


When selecting variables to investigate changes in the composition of the initially recruited sample over waves, care was taken to ensure that they represented the main publication domains, related to key survey items, and/or related to survey-specific motives for nonresponse (Schouten et al. 2011).

We included sociodemographic and socioeconomic variables (gender, age, education, citizenship, number of children, and income) in our models. Researchers have used these individual characteristics in almost all models for their substantive analyses based on SHARE data (SHARE-ERIC 2018). Additionally, some of these variables have been found to predict attrition in SHARE (Bristle et al. 2019). As Bristle et al. (2019) showed that item nonresponse to financial questions in SHARE negatively affected cooperation in the next wave, we supplemented the income quartiles with an additional category indicating that respondents did not answer the household income question.

We also included information on household composition, area of residence, employment status, and making ends meet, because this information has been widely used in economic research (SHARE-ERIC 2018) and has been found to predict cooperation in SHARE (Bristle et al. 2019). We included several key health variables that have been extensively used in the literature because researchers have also used SHARE data to study health (SHARE-ERIC 2018). Moreover, research has shown that persons with poor health tend to cooperate less than healthy persons (Bristle et al. 2019). Our selection of health variables included selfassessed health, chronic diseases, depression symptoms (Euro-D), limitations of instrumental activities of daily living (IADL), smoking and drinking behavior, and two objective health measurements/tests (grip strength and recall memory). As SHARE data are also used by researchers in the field of family and social networks, well-being, and charity, we included information on the spatial proximity of children, giving help to others, and receiving help from others. Additionally, as the literature shows that being socially active can predict cooperation in longitudinal studies (Bianchi and Biffignandi 2019), information on the number of social activities was also included.

Furthermore, research has shown that respondent burden in the previous SHARE wave influenced cooperation in the next wave (Bristle et al. 2019). In SHARE, selected household members serve as so-called family, financial, or household respondents and answer specific questions on behalf of the whole household. Being selected for one of these roles means that the duration of the interview is usually longer than average and that the respondent provides more information. To capture this respondent burden, we selected three interview process variables (financial, family, and household respondent).

\section{Evolution of the SHARE Panel Sample Over Waves and Across Countries}

\subsection{Analytical Approach}

Addressing Research Questions 1 and 2, we examined changes in the composition of the initially recruited SHARE sample over waves and across countries by calculating retention rates and estimating R-indicators for Analysis Samples A and B (the latter excludes reported deaths before the start of the corresponding wave and potentially includes unreported deaths). To investigate changes in the sample composition over waves, we 
coded participation for each wave. We denoted by $\mathrm{y}_{i}$ the outcome for respondent $i$ as follows:

$$
\mathrm{y}_{i}= \begin{cases}0 & \text { noparticipation } \\ 1 & \text { participation }\end{cases}
$$

where participation $\mathrm{y}_{i}$ equals 1 if respondent $i$ participated in the survey and 0 otherwise.

The retention rates in the present study measured the proportion of respondents who participated in each wave, conditional upon having participated in the first wave. The R-indicator (where " $R$ " stands for representativeness) was originally designed to measure the degree to which the respondents in a sample resemble the total target population or gross sample (Schouten et al. 2009). By contrast, the R-indicators in our study measured the degree to which the respondents in Analysis Sample A resemble the initially recruited first-wave respondents over waves, and the degree to which respondents in the dynamic Analysis Sample B resembles the initially recruited first-wave respondents over waves but excluding respondents who were reported to have died before a given wave.

Researchers have used R-indicators to assess the extent to which a net sample is representative of the target population or a gross sample. For instance, data of recruited samples have been compared with census, administrative, or population register data (e.g., Moore et al. 2016; Schouten et al. 2012; Luiten and Schouten 2013; Roberts et al. 2014). R-indicators can also be used as indicators for representativeness in panel studies (Schouten et al. 2012). Bianchi and Biffignandi (2017) used R-indicators to compare the panel sample of the UK household longitudinal study Understanding Society over waves with administrative data to assess population representativeness. In sum, they showed that $\mathrm{R}$-indicators were a valuable measure of representativeness.

R-indicators are estimated as follows (Schouten et al. 2009):

$$
\hat{R}_{\hat{\rho}}=1-2 \hat{S}_{\hat{\rho}}
$$

where $\hat{S}_{\hat{\rho}}$ is the estimated standard deviation of the individual response propensities. Therefore, the R-indicator is a measure of variation in response propensities. The estimated R-indicator $\hat{R}_{\hat{\rho}}$ ranges between 1 and 0 , where the value 1 denotes strong representativeness and the value 0 denotes the maximum deviation from strong representativeness.

Our approach differed from that of Schouten et al. (2009) with respect to the meaning of the term "representativeness." Schouten et al. (2009) designed R-indicators to assess the extent to which a net sample is representative of the total target population or a gross sample, whereas we used R-indicators to compare the composition of the initially recruited sample in Wave 1 of SHARE with the composition of the sample in subsequent waves, including any recruitment bias that might have existed in the original sample. The main advantage of our approach was that a rich set of individual-level data could be used rather than the sparse data that are available at population level. For our analyses of the evolution of the panel sample, all information already provided by the participants in the first wave could be used. This approach allowed for the detection of systematic dropout from the panel with respect to many important and substantive survey variables, and not only with respect to a few demographic variables available at the population level. 
Thus, we adapted Schouten and colleagues' concept (2009) to examine changes in the composition of the initially recruited SHARE sample over waves. We defined a panel response subset of variables $X$ as "fully representative" if the average propensity to participate again over these categories of $X$ was constant for all possible values of $X$ (Equation 2). For Analysis Sample A, samples in later waves were "fully representative" if their propensities to participate again were equal over the categories of $X$. As a consequence, the distributions of the selected respondent and household characteristics $X$ remained identical as in the first observed wave. For the dynamic Analysis Sample B, samples in later waves were "fully representative" if their propensities to participate again were equal over the categories of $X$ when reported deaths before a given wave were excluded. As a consequence, the distributions of the selected respondent and household characteristics $X$ remained identical as in the first observed wave excluding reported deaths before a given wave. The estimated R-indicator $\hat{R}_{\hat{\rho}}$ (Equation 2 ) in our study also ranged between 1 and 0 . However, 1 means no change in the composition of the original sample and 0 means total change. Confidence intervals for each $\mathrm{R}$-indicator in each wave were estimated at the five percent level.

The probability that the R-indicators would reach high values differed for our two analysis samples. We expected that the exclusion of reported deaths in the Analysis Samples B would lead to higher R-indicator values for the dynamic Analysis Sample B compared to the fixed Analysis Sample A because we assumed that respondents who dropped out because they died belonged to a selective group of respondents. In contrast, if we had perfect response or if we had equal response propensities over waves, the value of the R-indicator of both analysis samples (A and B) would remain at 1 .

To estimate the R-indicators, we used a specially adapted tool provided by the Representative Indicators for Survey Quality Project (RISQ 2015). In more detail, to compute R-indicators, we used a version of Version 2.1 of RISQ that was adapted for our purposes by the RISQ team. RISQ recommends that representativeness be analyzed by using categorical information rather than continuous information, we applied a categorical approach to describe and explore the evolution of the SHARE panel. We fitted several Rindicator models with the 26 selected variables based on participation outcome as the dependent variable. First, we estimated overall R-indicators for all countries (Analysis Sample A). Second, we estimated overall R-indicators that excluded reported deaths before a given wave for all countries (Analysis Sample B) to focus on non-mortalityrelated attrition. Third, we estimated the R-indicator based on Analysis Sample A and the R-indicator based on the dynamic Analysis Sample B for each country separately.

\subsection{Results}

To answer the first research question as to how the composition of the initially recruited first-wave sample evolved over waves, we calculated retention rates and estimated R-indicators for each wave, averaged across all countries.

The overall retention rate of Analysis Sample A declined almost linearly over the waves from $69 \%$ to $42 \%$ (Figure 1), with a kink at the first follow-up interview. Around $30 \%$ of the initially recruited first-wave respondents (Analysis Sample A) did not participate in the second wave. Also in the case of the R-indicator (Analysis Sample A), the largest decrease 
in the value was observed from the first to the second wave $(-.16)$. However, in contrast to the retention rate, the R-indicator (Analysis Sample A) decreased weakly over time afterwards. After six waves, Analysis Sample A reached an R-indicator value of .72. Thus, after the second wave, no further large decline in representativeness of the initially recruited first-wave sample and only few changes in the sample composition were observed.

Comparing the R-indicator for Analysis Sample A with that for Analysis Sample B, where we excluded reported deaths, we saw that the R-indicators of Analysis Sample B followed the same trend over waves as of Analysis Sample A - a substantial decrease in value after the first wave, and relatively stable values after the second wave. Moreover, we noted that the R-indicators for Analysis Sample B differed significantly from that of Analysis Sample A (see Figure 1). After six waves, the R-indicator for - and thus the representativeness of - Analysis Sample A was .72, whereas the R-indicator for the dynamic Analysis Sample B was .80. Thus, a decline in retention rate is not automatically linked to strong changes in the sample composition. In particular, when we eliminated the selective mortality-related attrition in Analysis Sample B, the representativeness of the sample was reasonably strong.

To answer Research Question 2 as to whether the evolution of the initially recruited sample over waves differed across countries, we calculated retention rates and estimated Rindicators for each country separately. Overall, the same pattern of declining retention rates and stabilizing R-indicators after the second wave was observed (Figure 2). Retention rates in Analysis Sample A ranged from 55\% to $75 \%$ across countries in Wave 2 and from $24 \%$ to $50 \%$ in Wave 6 . By contrast, the values of the $\mathrm{R}$-indicators in Wave 2 ranged across countries from .76 to .85 for Analysis Sample A and from .77 to .86 for Analysis Sample B. At the last observed wave (Wave 6), R-indicators ranged across countries from .61 to .74 for Analysis Sample A and from .69 to .85 for Analysis Sample B. Despite the fact that the gap between retention rates and R-indicators varied across countries, the observed pattern

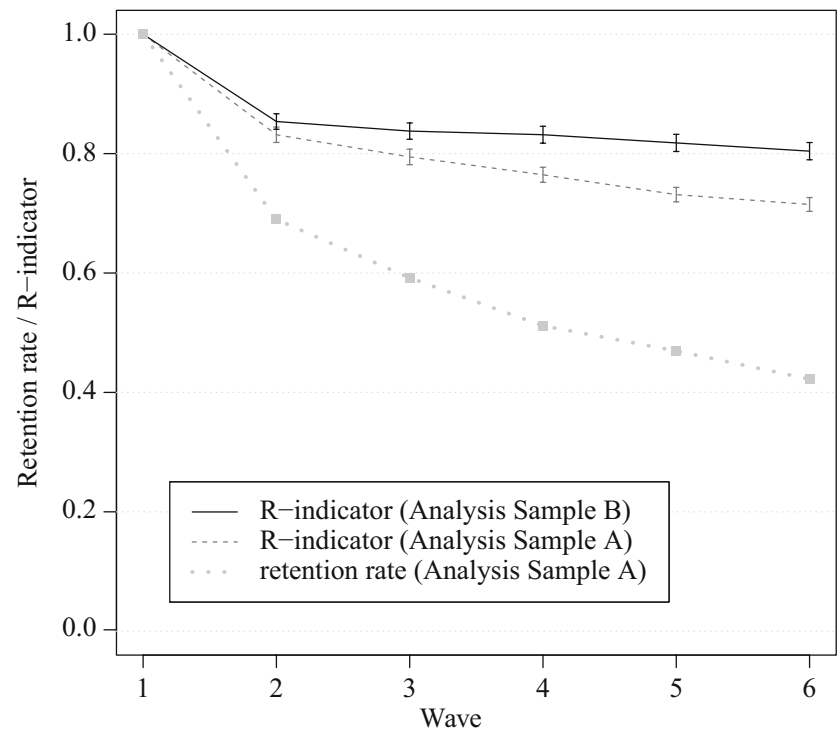

Fig. 1. Evolution of the initially recruited SHARE sample over waves. 

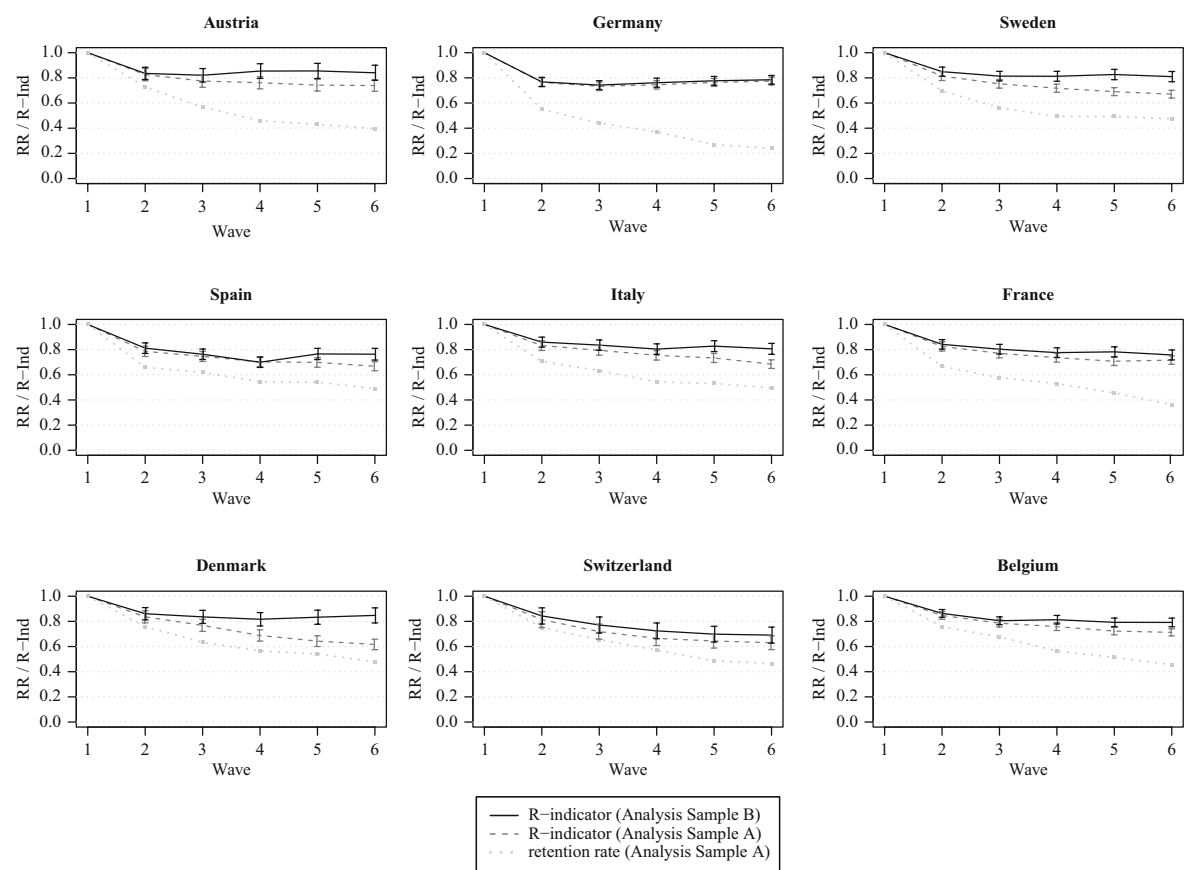

Fig. 2. Evolution of the initially recruited SHARE sample over waves, by country.

of change in the composition of the initially recruited first-wave sample (A and B) measured by R-indicators tended to be similar for all countries.

\section{Variable-Level Analysis of Non-Mortality-Related Attrition in SHARE}

\subsection{Analytical Approach}

Research Question 3 aims at understanding non-mortality-related attrition and asked what variables/characteristics played the most important role in the evolution of Analysis Sample B (which excludes reported deaths before a given wave) over waves across all countries. To answer this question, we calculated subgroup retention rates and estimated logistic regression models across all countries.

We defined several attrition scenarios for Research Question 3:

- Scenario 1 (W2): attrition in Wave 2,

- Scenario 2 (W3|W2): attrition in Wave 3, conditional upon participation in Wave 2,

- Scenario 4 (W6): attrition in Wave 6,

- Scenario 5 (W6|W3): attrition in Wave 6, conditional upon participation in Wave 4.

These scenarios will inform researchers about the changes in the composition of the initially recruited first-wave SHARE sample in later waves. For further exploration, we also defined and analyzed a number of other scenarios (see online Supplemental data, Table 1).

We compared subgroup retention rates for the defined scenarios with the first-wave subgroup proportions, excluding reported deaths before the given wave (dynamic Analysis 
Sample B). Only deviations of one percentage point or more are reported in the corresponding Figures (see Supplemental data, Figures 1-3), and only deviations of two percentage points or more are discussed in what follows.

In addition to the univariate subgroup retention rates, we explored non-mortality-related attrition within a multivariate framework because multivariate analyses allow several respondent and household characteristics to be taken into account at once. We estimated logit equations to examine which selected key variables have played the most important role in the evolution of the panel for the various selected scenarios. In contrast to the subgroup retention rates, the coding of $y_{i}$ was reversed intentionally for the multivariate logits. It allows for an interpretation of the results related to attrition rather than participation. Thus, the attrition propensity $\rho_{i}$ for a panel respondent $i$ is defined as follows:

$$
\rho_{i}(X)=P\left(y_{i}=1 \mid X=x_{i}\right) .
$$

For a respondent $i=1, \ldots, N, y_{i}$ refers to the binary nonresponse outcome, which equals 1 if panel respondent $i$ dropped out and 0 otherwise. The outcome $y_{i}$ can be different for each of the six waves; $x_{i}$ is a vector of the 26 selected SHARE key variables for panel respondent $i$ (Table 2).

As standard coefficients in logistic models indicate only the effect direction and provide no information about effect size, we estimated average marginal effects (AME) to evaluate the logistic regression coefficients more appropriately. AMEs represent the average change in probability when the variable predictor increases by one unit (Mood 2010). Moreover, by examining the $z$-scores of the logistic regression models we could quantify the impact of the individual and household characteristics on non-mortality-related attrition (Analysis Sample B). This examination deepened the understanding of which variables actually led to a decline of the R-indicators in Subsection 3.2.

\subsection{Results}

To answer Research Question 3 as to what variables/characteristics played the most important role in the changes in the composition of the initially recruited first-wave sample (Analysis Sample B, which excludes reported deaths before a given wave) over waves, we calculated subgroup retention rates on participation and ran logistic regression models on attrition for the selected scenarios.

\subsubsection{Wave 2}

In the subgroup retention rates in Wave 2, where respondents who were reported to have died before Wave 2 were excluded, we observed a deviation of two or more percentage points from the initially recruited Analysis Sample B in Wave 1 only for social activity (see online Supplemental data, Figure 1). The share of respondents who were socially active in Wave 1 increased by 2.4 percentage points in Wave 2, whereas the share of those who were not socially active increased by the same amount of percentage points.

The multivariate analyses of the Analysis Sample B in Wave 2, that excludes reported deaths before Wave 2, in Table 3 showed that, after controlling for other respondent and household characteristics, the association of being socially active with not participating in the second wave was statistically significant $(p<.001$; $z$-score $=-5.11$ ). The probability 
of attrition in Wave 2 decreased by four percentage points if respondents were socially active in Wave 1. However, the significant association of social activity with not participating in Wave 2 was not the strongest association observed. Rather, the strongest association of attrition in Wave 2 was observed with residing in a rural village $(p<.001$; $z$-score $=-9.12$ ). The probability of dropping out in Wave 2 was seven percentage points lower for respondents residing in rural villages than for those living in cities or large towns.

Other strong associations with attrition in Wave 2 were found for respondents who had participated in the grip strength test and who had reported their total household income in Wave 1 , regardless of the value in measure $(p<0.001 ; z$-scores between -3.75 and -5.93). They were less likely to drop out in Wave 2 than respondents who had not provided these measures. The decrease in probability to drop out ranged from five to eight percentage points (Table 3 ).

The multivariate analyses additionally showed that other numerous individual and household characteristics of Analysis Sample B in Wave 2 were significantly associated with attrition in the second wave (Table 3). The probability to drop out increased significantly with having received help from others, smoking, and having at least reported one limitation in IADL in the first wave. In addition to these positive significant associations with attrition in the second wave, we observed several negative significant associations with attrition in the second wave. Respondents who were between 60 and 69 years old in Wave 1 were less likely to drop out in Wave 2 than respondents who were between 50 and 59 years old in Wave 1. A respondent born in the survey country was less likely to attrite in Wave 2 than a respondent born outside the survey country. Highly educated respondents were less likely to attrite than low educated respondents, and respondents who resided in a small town in Wave 1 had a lower probability to drop out than respondents residing in a city or large town in Wave 1. Having children, among all groups of residential proximity of the child in Wave 1, decreased the probability to drop out in Wave 2 compared to having no children. Moreover, the probability to drop out in Wave 2 decreased significantly at the five percent level with giving help to others, having at least reported to have one chronic disease, having reported at least four depression symptoms, having a larger memory recall ability, and drinking, regardless of the frequency of alcohol consumption in the first wave.

\subsubsection{Wave 3}

The subgroup retention rates of Analysis Sample B in Wave 3, conditional upon participation in Wave 2, showed no deviations larger than two percentage points from the initially recruited respondents in the first wave when we excluded respondents that that were reported to have died before the third wave. Only one deviation larger than one percentage point was observed from respondents who resided in the city or large town. Their share was 1.1 percentage points lower compared to their share in Wave 1 (result not shown).

Multivariate analyses showed that strong predictors of attrition in Wave 3, conditional upon participation in Wave 2, were: high educational level $(p<.001 ; z$-score $=-4.98)$ compared to a low educational level, social activity in Wave $1(p<.001 ; z$-score $=-3.92)$, age between 60 and 69 years in Wave $1(p<.001 ; z$-score $=-3.48)$ compared to age between 50 and 59 years in Wave 1 , birth in survey country level $(p<.001$; 
Table 3. Estimated average marginal effects (AME) from logistic regressions of attrition by individual and household characteristics.

W2 W3IW2 W6 W6IW3

Gender: male (ref.)

- female

$$
\begin{array}{cccc}
-.01 & -.01 & -.04 * * & -.01 \\
(-1.15) & (-.46) & (-2.96) & (-.99)
\end{array}
$$

Age: 50-59 years (ref.)

- 60-69 years

$\begin{array}{cccc}-.02 * & -.04 * * * & -.03 * & -.00 \\ (-2.23) & (-3.48) & (-2.38) & (-.38) \\ .00 & -.03 * & -.01 & .01 \\ (.06) & (-2.11) & (-.72) & (.58) \\ -.00 & .01 & .11 * * * & .10 * * * \\ (-.16) & (.81) & (4.78) & (3.42)\end{array}$

Born in survey country: no (ref.)

- yes

$$
\begin{array}{cccc}
-.05 * * * & -.05 * * * & -.09 * * * & -.07 * * * \\
(-3.86) & (-3.38) & (-6.27) & (-4.05)
\end{array}
$$

Education level: low (ref.)

- medium

- high

$$
\begin{array}{cc}
-.00 & -.02 \\
(-.51) & (-1.81) \\
-.04 * * * & -.05 * * * \\
(-4.14) & (-4.98)
\end{array}
$$

$$
\begin{gathered}
-.01 \\
(-1.00)
\end{gathered}
$$$$
-.01
$$$$
(-.46)
$$$$
-.07 * * *
$$

HH size: 1-person (ref.)

- 2-person $\mathrm{HH}$

$$
.00
$$

- 3+ person $\mathrm{HH}$

.02

(.92)

$(-6.43)$

$(-4.20)$

Partner in HH: no (ref.)

- yes

$$
.01
$$

Area of residence: city/large town (ref.)

- small town

- rural village

$$
\begin{array}{cc}
-.03 * * * & -.03 * * \\
(-4.26) & (-3.08) \\
-.07 * * * & -.03 * * \\
(-9.12) & (-3.00)
\end{array}
$$

(1.26)

.02

$$
\text { (.76) }
$$

\section{Residential proximity of child(ren):}

$$
.02-01
$$
no children (ref.)

- child in $\mathrm{HH}$

- child $=1 \mathrm{~km}$ away

$$
\begin{array}{cc}
-.08 * * * & -.03 \\
(-5.61) & (-1.69) \\
-.06 * * * & -.01 \\
(-4.39) & (-.72) \\
-.04 * * * & -.01 \\
(-3.95) & (-1.10)
\end{array}
$$$$
-.05 * * *
$$$$
-.05 * * *
$$$$
(-5.56)
$$$$
-.07 * * *
$$$$
-.03 *
$$$$
(-6.90)
$$$$
(-2.54)
$$

- child $>1 \mathrm{~km}$ away

$$
\begin{gathered}
-.07 * * * \\
(-4.23) \\
-.08 * * * \\
(-5.04) \\
-.05 * * * \\
(-3.86)
\end{gathered}
$$

$$
\begin{array}{r}
-.04 * \\
(-2.33)
\end{array}
$$$$
-.04 *
$$$$
(-2.03)
$$$$
-.02
$$$$
(-1.17)
$$

Social activities: no activities (ref.)

- at least one activity

$$
\begin{array}{ll}
-.04 * * * & -.03 * * * \\
(-5.11) & (-3.92)
\end{array}
$$$$
-.06 * * *
$$$$
-.04 * * *
$$$$
(-7.06)
$$$$
(-4.12)
$$

\section{Received help from others: no (ref.)}

- yes

$$
\begin{array}{cccc}
.02 * & -.02 * & -.02 & -.02 \\
(2.08) & (-2.06) & (-1.86) & (-1.66)
\end{array}
$$

Gave help to others: no (ref.)

$$
\begin{array}{lcccc}
- \text { yes } & -.02 * & -.01 & -.01 & -.00 \\
& (-2.44) & (-1.86) & (-.74) & (-.06)
\end{array}
$$


Table 3. Continued

$\begin{array}{lll}\text { W2 W3IW2 W6 } & \text { W6IW3 }\end{array}$

Health status: good/better (ref.)

- poor or fair

.01

.02

.02

Chronic diseases: none (ref.)

$-1+$ chronic diseases

$$
\begin{array}{cccc}
-.02 * & -.02 * & -.01 & -.02 * \\
(-2.25) & (-2.22) & (-1.32) & (-2.25)
\end{array}
$$

Depression (Euro-D): insufficient symptoms (ref.)

- 4+ symptoms

$$
\begin{array}{llrc}
-.02 * * & -.00 & -.02 * & -.01 \\
(-2.96) & (-.39) & (-2.33) & (-.57)
\end{array}
$$

Maximum grip strength: item nonresponse (ref.)

$-1^{\text {st }}$ quartile (very weak)

$$
\begin{array}{cccc}
-.08 * * * & -.01 & -.04 & -.02 \\
(-4.84) & (-.71) & (-2.65) & (-.68) \\
-.07 * * * & -.01 & -.06 * * & .01 \\
(-4.60) & (-.29) & (-1.85) & (.50) \\
-.08 * * * & -.00 & -.07 * * & -.01 \\
(-4.73) & (-.05) & (-3.20) & (-.33) \\
-.08 * * * & .00 & -.04 & -.00 \\
(-4.52) & (.10) & (-3.08) & (-.18)
\end{array}
$$

$-2^{\text {nd }}$ quartile

$-3^{\text {rd }}$ quartile

$-4^{\text {th }}$ quartile (very strong)

\section{Memory recall ability:}

- less than half of the words (ref.)

- more than half of the words

$$
\begin{array}{cccc}
-.03 * * * & -.01 & -.02 * * & -.02 \\
(-4.27) & (-1.66) & (-2.62) & (-1.84)
\end{array}
$$

Hospital overnight stay in last 12 months: no (ref.)

- yes

Currently smoking: no (ref.)

$$
- \text { yes }
$$

Currently drinking: never (ref.)

- less than once a week

- 1-6 times a week

- almost every day

IADL: no IADL limitations (ref.)

- 1+ IADL limitations

Employment status: retired (ref.)

- working

- not working and other

Making ends meet: difficulties (ref.)

- no difficulties

$$
\begin{array}{llll}
-.00 & -.00 & -.00 & -.01 \\
(-.39) & (-.01) & (-.40) & (-.80)
\end{array}
$$

$$
.03 * * *
$$

$$
\text { (3.95) }
$$

$.04 * * *$

(4.43)

$$
\begin{array}{cc}
-.03 * * & -.02 \\
(-2.70) & (-1.65) \\
-.04 * * * & -.02 \\
(-4.07) & (-1.65) \\
-.04 * * * & -.01 \\
(-4.33) & (-.89)
\end{array}
$$

$-.05 * * *$

$(-3.81)$

$-.05 * * *$

$(-4.68)$

$-.04 * * *$

$(-3.77)$

$(-2.41)$

$-.05 * * *$

$$
.03 * *
$$

$.03 *$

(2.16)

$$
.04 * * *
$$

$(-1.62)$

$$
\text { (3.03) }
$$$$
-.00
$$

$$
\begin{gathered}
.01 \\
(.97)
\end{gathered}
$$$$
(-.03)
$$

$-.00$

$-.00$

$(-.34)$

$(-.47)$

$$
\begin{array}{cc}
-.01 & -.00 \\
-1.65) & (-.13)
\end{array}
$$

$$
\begin{gathered}
-.01 \\
(-.95)
\end{gathered}
$$

$-.01$

$.04 * * * \quad-.02$

(4.43) (-1.67) 
Table 3. Continued

\section{W2 W3IW2 W6 W6IW3}

Total household income: item nonresponse (ref.)

- 1st quartile

$-.07 * * *$

- 2nd quartile

$(-5.54)$

$-.04 * *$

$-.05 * * *$

$-.04 *$

$-5.54)$

$(-3.08)$

$(-3.39)$

$(-2.11)$

$(-5.93)$

$-.05 * * *$

$-.06 * * *$

$-.04 *$

-3 rd quartile

$\begin{array}{ll}-.06 * * * & (-3.37) \\ -.06 * * *\end{array}$

$(-4.51)$

$(-2.49)$

$(-4.92)$

- 4th quartile

$-.05 * * * \quad-.04 * *$

$-.06 * * *$

$-.05 * * * \quad-.04 *$

$(-4.64)$

$(-1.51)$

$(-3.75) \quad(-2.83)$

$-.04 * *$

$-.00$

Family respondent: no (ref.)

- yes

$\begin{array}{cccc}-.01 & -.00 & -.01 & .01 \\ (-.92) & (-.19) & (.62) & (.80) \\ & & & \\ .02 & -.00 & -.01 & -.02 \\ (1.25) & (-.04) & (-.95) & (-1.27)\end{array}$

Financial respondent: no (ref.)

$$
\text { - yes }
$$

Household respondent: no (ref.)

- yes

$$
\begin{array}{llll}
-.03 & -.00 & -.01 & -.00
\end{array}
$$

$\begin{array}{llll}(-1.95) & (-.03) \quad(-.57) \quad(-.08)\end{array}$

\begin{tabular}{lllll}
\hline $\mathrm{N}$ & 19,761 & 13,466 & 16,619 & 10,412 \\
\hline
\end{tabular}

Note. W2 = attrition in Wave 2; W3|W2 = attrition in Wave 3, conditional upon participation in Wave 2 ; $\mathrm{W} 6=$ attrition in Wave 6; W6|W3 = attrition in Wave 6, conditional upon participation in Wave 3. $\mathrm{Z}$ statistics in parentheses. $\mathrm{HH}=$ Household; IADL $=$ instrumental activities of daily living; all models additionally include country dummies; ${ }^{*} p<.05,{ }^{* *} p<.01,{ }^{* * *} p<.001$.

$z$-score $=-3.38$ ), and reporting the total household income among all income groups $(p<.01 ; z$-scores between -2.83 and -4.21$)$ compared to item nonresponse in the total household income in Wave 1 (Table 3).

Other significant negative associations with attrition were observed for respondents who were between 70 and 79 years old, resided in a small town or rural village, received help from others, and reported at least one chronic disease in the first wave compared to corresponding reference category. Other positive significant associations with attrition in the third wave were observed with having reported at least one IADL limitation in Wave 1 (Table 3).

Some significant effects of individual and household charactersitics on attrition we found in the model for the second wave, that excluded reported deaths before Wave 2, could not be found in the conditional model for the third wave, where we excluded reported deaths before Wave 3 (Table 3).

\subsubsection{Wave 6}

The proportion of respondents who were born in the survey country, and of respondents who self-assessed their health in Wave 1 as good or better, and of respondents who were socially active in Wave 1 was between 2.40 and 3.64 percentage points larger for the panel members who participated in Wave 6 compared to the respective Wave 1 proportions. Moreover, the proportion of respondents who had a medium educational level was 3.06 percentage points smaller compared to the respective Wave 1 proportion (see 
Supplemental data, Figure 2). In the conditional Wave 6 scenario (attrition in Wave 6 , conditional upon participation in Wave 3) no larger deviation than two percentage points were observed (see Supplemental data, Figure 3).

Examining multivariate attrition in Wave 6, we observed for the unconditional scenario that many individual and household characteristics significantly predicted the drop out in the sixth wave (Table 3). Strong positive associations with attrition were found for respondents who smoked $(p<.001 ; z$-score $=4.43)$, made ends meet with no difficulties $(p<.001 ; z$-score $=4.43)$, and reported at least one IADL limitation in the first wave $(p<.001 ; z$-score $=3.41)$ compared to respondents who did not smoke, made ends meet with difficulties, and reported no IADL limitation in the first wave. The probability to drop out increased by four percentage points for each of these characteristics (smoking, making ends meet, and having at least one IADL limitation). Strong negative associations with attrition were found for respondents who were socially active $(p<.001$; $z$-score $=-7.06)$, had a high educational level $(p<.001 ; z$-score $=-6.43)$ compared to low educational level, were born in survey country $(p<.001 ; z$-score $=-6.27)$, resided in a rural village $(p<.001 ; z$-score $=-6.90)$ or small town $(p<.001$; $z$-score $=-5.56$ ) compared to city or large town. The decrease in probability to drop out for these groups ranged between four and ten percentage points. For further negative and positive associations in Wave 6 (with a lower significance level than $99.9 \%$ or with a smaller absolute value in $z$-score than 5) please see Table 3.

For attrition in Wave 6, conditional upon participation in Wave 3, we observed at the significance level of $99.9 \%$, that highly educated and socially active respondents in Wave 1 , and who were born in the survey country were less likely to drop out in Wave 6 than low-educated and socially inactive respondents and those, who were born outside the survey country (Table 3). Furthermore, residing in a small town and drinking between one and six drinks peer week, compared to residing in a city or large town and not drinking in Wave 1 decreased the probability of dropping out by five percentage points for the respective characteristics (Table 3). For further negative and positive associations (with a lower significance level than $99.9 \%$ ) please see Table 3.

Comparing the conditional Wave 6 attrition model with the unconditional Wave 6 attrition model, we noted that far fewer individual and household characteristics were significantly associated with attrition in the conditional model. However, the age group $80+$ in Wave 1 , who were aged $92+$ years in Wave 6 , had a relatively large positive impact in both Wave 6 attrition models. The probability to drop out increased by eleven percentage points in the unconditional model and by ten percentage points in the conditional model for those old respondents (Table 3).

\section{Summary and Discussion}

This study examined the evolution of the initially recruited SHARE first-wave sample. With its specific target population, SHARE has a relatively large proportion of respondents who are at a high risk of attrition because of death. As we assumed that people who die are a selective group of the population and of the panel sample, we investigated the evolution of the SHARE panel with two defined samples. We used Analysis Sample A to study total attrition (non-mortality-related and mortality-related attrition), and Analysis 
Sample B to study exclusively non-mortality-related attrition. We applied different methods to answer our research questions.

We answered Research Question 1 "How has the initially recruited SHARE first-wave sample (A and B) has evolved over waves" by calculating retention rates and estimating Rindicators. We detected declining retention rates with a major loss of respondents in the second wave. This finding is in line with previous literature (Lepkowski and Couper 2002; Schoeni et al. 2013; Lugtig 2014). Moreover, the retention rates observed in our study are about the same as those for second-wave response in other studies of older populations (Banks et al. 2011). In addition, we observed that the values of the R-indicators of the initially recruited SHARE sample (Analysis Sample A and B) dropped in the second wave but remained stable afterwards. Thus, we could show that, despite declining retention rates, the composition of the first-wave sample changed, but was maintained over waves with respect to many individual or household characteristics after the second wave. Furthermore, the results showed, when we excluded respondents that had been reported as dead before a given wave (Analysis Sample B, Wave 1 - Wave 6), that, the observed changes in the sample composition over time were mainly due to deaths (with the exception of Wave 2).

As SHARE collects data in various countries, it has to deal with country-specific differences, although it is harmonized ex ante. Therefore, we further investigated the evolution of the SHARE panel by Research Question 2 "Has the evolution of the initially recruited first-wave sample (A and B) over waves varied across countries?". We observed that the changes in the composition of the initially recruited sample over time differed across countries, although the differences were small. All countries followed the same trend, with a stable R-indicator value after the second wave (Analysis Samples A and B). However, comparing R-indicator values for Analysis Sample B (excluding deaths before a given wave) revealed larger differences across countries. These differences may be due to the quality of the respective death reports.

To answer Research Question 3 as to what characteristics and variables played the most important role in the changes in the composition of the initially recruited first-wave sample (dynamic Analysis Sample B) over waves, we examined various attrition scenarios by calculating subgroup retention rates and estimating multivariate logistic regression models on attrition. The results of the subgroup retention rate analyses were supported by those of the multivariate analyses. In all multivariate models, first-wave respondents who were born in the survey country, were residing in a rural area or small town, had a high level of education, and were socially active were less likely to attrite than first-wave respondents who were not born in the survey country, who were residing in a city or a large town, who had a low level of education, and were socially inactive. We did not observe that healthrelated variables, such as illness or age, were strong predictors of non-mortality-related attrition. Only very old respondents (aged $80+$ in the first wave) had a high risk of attrition in later waves. Overall, birth in survey country, area of residence, education, and social activities played an important role in the non-mortality related attrition and their impact led to a decline of the R-indicators.

Comparing logit models from early waves with those from later waves, we noted that some significant associations declined to statistical insignificance in the multivariate models, especially in the models for attrition conditional upon participation in a specified 
previous wave. This change in significance is reflected in the stabilizing R-indicator after the second wave.

The present study has a number of limitations. To draw conclusions from panel data about the general population aged 50 years or older, researchers need to consider and investigate initial nonresponse - that is, nonresponse that occurs in the recruitment stage of the panel. As the focus of the present study was on the evolution of the initially recruited first-wave sample over waves, we did not consider initial nonresponse. However, as initial nonresponse is an important factor for understanding the overall nonresponse process in SHARE and might have an impact on the data researchers use for analyzing dynamic processes in the European societies, future research should take it into account.

Another limitation of this study relates to the reporting of deaths. The SHARE countries included in the study differed in the share of reported deaths in the initially recruited sample over the course of the panel. Unlike the U.S. Health and Retirement Survey (HRS) or the English Longitudinal Survey of Ageing (ELSA) in England, SHARE cannot be linked to a mortality register because national mortality registers are lacking in most European countries (Bergmann et al. 2019). A comparison of the share of reported deaths in the initially recruited first-wave sample in SHARE with the mortality rate among persons aged 50+ years between 2004 and 2015 in Eurostat data (Eurostat 2004-2015) showed that only in a minority of the SHARE countries in our study was the share of respondents who died over the course of the panel lower than the estimated share of persons in the corresponding population group who died between 2004 and 2015 (Supplement data, Table 2). Thus, we may have underestimated the number of deaths in SHARE for a few countries due to a lack of information. However, we expected the share of deaths in Eurostat and SHARE to differ to some extent because SHARE excludes the hospitalized population from the sampling frame.

Notwithstanding these limitations, our study shows that, despite declining retention rates, the composition of an initially recruited panel sample can remain stable over later waves. The representativeness of the first wave sample (fixed Analysis Sample A and dynamic Analysis Sample B) did not decline further after the second wave. Moreover, this study informs researchers who wish to analyze dynamic processes over time about the impact of mortality-related and non-mortality-related attrition on the composition of the initially recruited first-wave SHARE sample over time. To further inform researchers wishing to analyze dynamic processes in SHARE over time, future research should examine the impact of mortality- and non-mortality-related attrition on cross-sectional and longitudinal estimates.

\section{References}

AAPOR (American Association for Public Opinion Research). 2016. Standard Definitions: Final Dispositions of Case Codes and Outcome Rates for Surveys (9th ed.). AAPOR. Available at: https://www.aapor.org/AAPOR_Main/media/publications/ Standard-Definitions20169theditionfinal.pdf (accessed August 2018).

Banks, J., M. Alastair, and J.P. Smith. 2011. "Attrition and Health in Ageing Studies: Evidence from ELSA and HRS." Longitudinal and Life Course Studies 2: 1-29. DOI: https://doi.org/10.14301/llcs.v2i2.115. 
Bergmann, M., T. Kneip, G. De Luca, and A. Scherpenzeel. 2019. Survey Participation in the Survey of Health, Ageing and Retirement in Europe (SHARE), Wave 1-7. SHARE Working Paper Series 31-2017. Munich, Germany: Munich Center for the Economics of Aging (MEA), Max Planck Institute for Social Law and Social Policy. Available at: http://www.share-project.org/uploads/tx_sharepublications/WP_Series_41_2019_ Bergmann_et_al.pdf (accessed February 2019).

Bianchi, A. and S. Biffignandi. 2017. "Representativeness in Panel Surveys." Mathematical Population Studies 24: 126-143. DOI: https://doi.org/10.1080/ 08898480.2016.1271650.

Bianchi, A. and S. Biffignandi. 2019. "Social Indicators to Explain Response in Longitudinal Studies.” Social Indicators Research 141: 931-957. DOI: https://doi.org/ 10.1007/s11205-018-1874-7.

Binder, D. 1998. "Longitudinal Surveys: Why Are These Surveys Different from All Other Surveys?” Survey Methodology 24: 101-108. Available at: https://www150. statcan.gc.ca/n1/en/catalogue/12-001-X19980024347 (accessed June 2020).

Börsch-Supan, A., M. Brandt, C. Hunkler, T. Kneip, J. Korbmacher, F. Malter, B. Schaan, S. Stuck, and S. Zuber. 2013. "Data Resource Profile: The Survey of Health, Ageing and Retirement in Europe (SHARE)." International Journal of Epidemiology 42: 992-1001. DOI: https://doi.org/10.1093/ije/dyt088.

Börsch-Supan, A. 2017. Survey of Health, Ageing and Retirement in Europe (SHARE) Wave 1. Release version: 6.0.0. SHARE-ERIC. DOI: https://doi.org/10.6103/SHARE. w1.600.

Bristle, J., M. Celidoni, C. Dal Bianco, and G. Weber. 2019. "The Contributions of Paradata and Features of Respondents, Interviewers and Survey Agencies to Panel Co-Operation in the Survey of Health, Ageing and Retirement in Europe." Journal of the Royal Statistical Society: Series A (Statistics in Society) 182: 3-35. DOI: https://doi. org/10.1111/rssa.12391.

Buck, N., J. Burton, H. Laurie, P. Lynn, and S.C.N. Uhrig. 2006. Quality Profile: British Household Panel. Survey Version 2.0: Waves 1 to 13: 1991-2003. Colchester, UK: University of Essex, Institute for Social and Economic Research.

De Luca, G., C. Rossetti, and F. Malter. 2013. "Sample Design and Weighting Strategies in SHARE Wave 5." In SHARE Wave 5: Innovations \& Methodology, edited by F. Malter and A. Börsch-Supan, 75-84. Munich, Germany: Munich Center for the Economics of Aging, Max Planck Institute for Social Law and Social Policy.

Eurostat. 2004-2015. Available at: "https://ec.europa.eu/eurostat/web/population-demography-migration-projections/population-projections-/database (accessed June 2019).

Fitzgerald, J., P. Gottschalk, and R. Moffitt. 1998. "An Analysis of Sample Attrition in Panel Data: The Michigan Panel Study of Income Dynamics.” The Journal of Human Resources 33: 251-299. DOI: https://doi.org/10.2307/146433.

Leon, D.A. 2011. "Trends in European life expectancy: a salutary view." International Journal of Epidemiology 40: 271-77. DOI: https://doi.org/10.1093/ije/dyr061.

Lepkowski, J.M. and M.P. Couper. 2002. "Nonresponse in the Second Wave of Longitudinal Household Surveys." In Survey Nonresponse, edited by R.M. Groves, D.A. Dillman, J.L. Eltinge, and R. J.A. Little, 259-272. New York: John Wiley \& Sons. 
Lugtig, P. 2014. "Panel Attrition.” Sociological Methods \& Research 43: 699-723. DOI: https://doi.org/10.1177/0049124113520305.

Luiten, A. and B. Schouten. 2013. "Tailored fieldwork design to increase representative household survey response: an experiment in the Survey of Consumer Satisfaction." Journal of the Royal Statistical Society: Series A (Statistics in Society) 176: 169-189. DOI: https://doi.org/10.1111/j.1467-985X.2012.01080.x.

Lynn, P. 2009. "Methods for Longitudinal Surveys." In Methodology of Longitudinal Surveys, edited by P. Lynn, 1-19. Chichester, UK: John Wiley \& Sons.

Lynn, P. and P. Lugtig. 2017. "Total Survey Error for Longitudinal Surveys.” In Total Survey Error in Practice, edited by P.P. Biemer, E. De Leeuw, S. Eckman, B. Edwards, F. Kreuter, L.E. Lyberg, N.C. Tucker, and B.T. West, 279-298. Chichester, UK: John Wiley \& Sons.

Mood, C. 2010. "Logistic Regression: Why We Cannot Do what We Think We Can Do, and What We Can Do about It." European Sociological Review 26: 67-82.

Moore, J.C., G.B. Durrant, and P.W. F. Smith. 2016. "Data Set Representativeness during Data Collection in Three UK Social Surveys: Generalizability and the Effects of Auxiliary Covariate Choice." Journal of the Royal Statistical Society: Series A (Statistics in Society): 229-248. DOI: https://doi.org/10.1111/rssa.12256.

Myrskylä, M., J.R. Goldstein, and Y.A. Cheng. 2013. "New Cohort Fertility Forecasts for the Developed World: Rises, Falls, and Reversals." Population and Development Review 39: 31-56. DOI: https://doi.org/10.1111/j.1728-4457.2013.00572.x.

Olsen, R.J. 2018. "Respondent Attrition Versus Data Attrition and Their Reduction." In The Palgrave Handbook of Survey Research, edited by D.L. Vannette and J.A. Krosnick, 155-158. Cham, Switzerland: Springer.

RISQ. 2015. Representative Indicators for Survey Quality-Tools. Manchester, UK: University of Manchester. Available at: https://www.cmist.manchester.ac.uk/research/projects/representative-indicators-for-survey-quality/tools/ (accessed August 2018).

Roberts, C., C. Vandenplas, and M.E. Stähli. 2014. "Evaluating the Impact of Response Enhancement Methods on the Risk of Nonresponse Bias and Survey Costs." Survey Research Methods 8: 67-80. DOI: https://doi.org/10.18148/srm/2014.v8i2.5459.

Schoeni, R.F., F. Stafford, K.A. McGonagle, and P. Andreski. 2013. "Response Rates in National Panel Surveys." Annals of the American Academy of Political and Social Science 645: 60-87.

Schouten, B., J. Bethlehem, K. Beullens, Ø. Kleven, G. Loosveldt, A. Luiten, Ka. Rutar, N. Shlomo, and C. Skinner. 2012. "Evaluating, Comparing, Monitoring, and Improving Representativeness of Survey Response through R-Indicators and Partial R-Indicators." International Statistical Review 80: 382-399. DOI: https://doi.org/10.1111/j.17515823.2012.00189.x.

Schouten, B., F. Cobben, and J. Bethlehem. 2009. "Indicators for the Representativeness of Survey Response.” Survey Methodology 35: 101-113.

Schouten, B., N. Shlomo, and C. Skinner. 2011. "Indicators for Monitoring and Improving Representativeness of Response.” Journal of Official Statistics 27: 1-24. Available at: https://www.scb.se/contentassets/ca21 efb41fee47d293bbee5bf7be7fb3/indicators-formonitoring-and-improving-representativeness-of-response.pdf (accessed May 2020). 
SHARE-ERIC. 2018. SHARE Publications: Journal Articles.Available at: http://www. share-project.org/share-publications/journalarticles00.html (accessed August 2018).

Smith, P., P. Lynn, and D. Elliot. 2009. "Sample Design for Longitudinal Surveys." In Methodology of Longitudinal Surveys, edited by M. Groves, G. Kalton, J.N. Rao, N. Schwarz, C. Skinner, and P. Lynn, 21-33. Chichester, UK: John Wiley \& Sons.

Watson, D. 2003. "Sample Attrition between Waves 1 and 5 in the European Community Household Panel." European Sociological Review 19: 361-378. DOI: https://doi.org/ 10.1093/esr/19.4.361.

Watson, N. and M. Wooden. 2009. "Identifying Factors Affecting Longitudinal Survey Response.” In Methodology of Longitudinal Surveys, edited by P. Lynn, 157-181. Chichester, UK: John Wiley \& Sons.

Watson, N. and M. Wooden. 2019. "Chasing Hard-to-Get Cases in Panel Surveys: Is it Worth it?" methods, data, analyses 13: 199-222. DOI: https://doi.org/10.12758/ mda.2018.03.

Received August 2020

Revised June 2019

Accepted February 2020 